\title{
Health-related Quality of Life and Satisfaction of the Patient Following Orthognathic Surgery in Kingdom of Saudi Arabia
}

\author{
Ghadi DA Duhduh ${ }^{1}$, Tasneem KM Arishi ${ }^{2}$, Omar A Darraj ${ }^{3}$, Aziza MA Alqadi ${ }^{4}$
}

\begin{abstract}
Introduction: Most patients undergo orthognathic surgery (OS) to improve their beauty and correct functional imbalances. OS is also performed in the case of temporomandibular joint disorders to reduce facial pain and enhance the quality of life of patients.

Aim: The aim of the study was to evaluate the quality of life of patients who undergo OS in terms of esthetic appearances, chewing, and postoperative complications and to assess the percentage of satisfaction among patients in terms of facial appearances, friends' and relatives' opinions, and functional performance of the oral cavity.

Materials and methods: Data of 73 patients who underwent OS in Kingdom of Saudi Arabia were collected from their medical records. A brief questionnaire was prepared on the basis of previous studies with some modifications. Data were collected and a questionnaire was answered via a telephone interview with these patients. Questions were related to the patients' basic demographic information and their postoperative complications, such as swelling, pain, chewing difficulty, and snoring. The second part was introduced as a questionnaire designed by the researchers to measure the patients' satisfaction regarding OS. This questionnaire was related to the appearance of the face from the side and front views and to the level of attractiveness perceived by patients themselves and their relatives and friends after OS. This questionnaire also included improvements in oral functions, such as chewing. The overall performance of the oral cavity was assessed. Data were analyzed using SPSS. Results: A total of 50 patients completed the telephone interview and the examination. The highest number of subjects was in the young age group of 19-25 years. Of these patients, 31 (62\%) showed a positive result on the snoring outcomes, and $25(50 \%)$ complained of pain and swelling. Furthermore, $33(66 \%)$ and 27 (54\%) patients reported improvements in chewing and facial esthetic outcomes, respectively. More than $50 \%$ of the patients were satisfied in their facial appearance from the side and front views. Patients' perception of their attractiveness and their friends' and family's opinion about their facial appearance recorded were between $60 \%$ and $62 \%$. Other questions dealing with improvements in eating, chewing, and overall oral cavity performance gained $42-18 \%$. All variables showed significant differences at the level of $p$ value more than 0.050 . Conclusion: Overall, the majority of the OS patients showed positive results in terms of chewing, snoring, facial esthetics, pain, and swelling during their recovery time. Patients are mostly satisfied with the overall facial attractiveness results as well as the opinions of their relatives and friends. Clinical significance: OS is performed to enhance the appearance and function of the oral cavity, which may end with a positive effect of the patient's oral function, self-esteem, and social interaction.
\end{abstract}

Keywords: Facial esthetic, Orthognathic surgery, Postoperative complication, Quality of life.

World Journal of Dentistry (2019): 10.5005/jp-journals-10015-1646

\section{INTRODUCTION}

Orthognathic surgery (OS), including a number of surgical procedures performed on jaws and chin, is conducted to correct the relationship between dental arches and teeth. ' If the foundation of the maxillary and mandibular jaws is disrupted, OS is performed to enhance the appearance and function of the oral cavity. ${ }^{2}$

The disfigurement of the face may negatively affect the social interaction of patients. ${ }^{3}$ Most patients undergo OS to improve their beauty and correct functional imbalances. Various studies have revealed that OS improves the self-confidence and social skills of patients. ${ }^{4}$ Facial appearance and beauty also enhance the psychological condition of patients. ${ }^{5}$ OS is performed in the case of temporomandibular joint disorders to reduce facial pain and improve the quality of life of patients. ${ }^{6}$

Traditional OS involves preoperative orthodontic treatments, OS, and postoperative orthodontic treatments. Other procedures, such as first-approach surgery, have been introduced to overcome the disadvantages of traditional OS. ${ }^{7}$ OS has been considered a safe and generally effective medical treatment and has been preferred by patients to improve their facial appearance and functional outcomes. $^{8}$

In addition to these positive aspects of OS, some risks (such as temporary or permanent nerve damage, malunion fracture,
1,2,4 Private Practice, Jazan, Kingdom of Saudi Arabia

${ }^{3}$ Intern Department, College of Dentistry, Jazan University, Jazan, Kingdom of Saudi Arabia

Corresponding Author: Omar A Darraj, Intern Department, College of Dentistry, Jazan University, Jazan, Kingdom of Saudi Arabia, Phone: +966 552258123, e-mail: dr.o.darraj@gmail.com

How to cite this article: Duhduh GDA, Arishi TKM, Darraj OA, et al. Health-related Quality of Life and Satisfaction of the Patient Following Orthognathic Surgery in Kingdom of Saudi Arabia. World J Dent 2019;10(4):270-274.

Source of support: Nil

Conflict of interest: None

nonunion fracture, infections, TMJ issues, tooth necrosis, and pain management) are associated with this surgery during and after OS. ${ }^{9}$ Therefore, post-surgical swelling and pain during the recovery time of patients should be managed. ${ }^{10,11}$

Few studies have been conducted in Kingdom of Saudi Arabia. For example, Zahrani ${ }^{12}$ evaluated the satisfaction or dissatisfaction of patients' perception of the esthetic and functional outcomes of OS. Studies have evaluated hard tissue cephalometric and different necessary ethnic facial norms to properly plan OS for a sample of

o The Author(s). 2019 Open Access This article is distributed under the terms of the Creative Commons Attribution 4.0 International License (https://creativecommons. org/licenses/by-nc/4.0/), which permits unrestricted use, distribution, and non-commercial reproduction in any medium, provided you give appropriate credit to the original author(s) and the source, provide a link to the Creative Commons license, and indicate if changes were made. The Creative Commons Public Domain Dedication waiver (http://creativecommons.org/publicdomain/zero/1.0/) applies to the data made available in this article, unless otherwise stated. 
adults in Kingdom of Saudi Arabia. ${ }^{13,14}$ Abdullah $^{15}$ measured the effect of OS on the quality of life in patients in Kingdom of Saudi Arabia. Al Asseri ${ }^{16}$ evaluated the spectrum and characteristics of all patients treated with OS in four main medical centers in Riyadh and explored the common types of OS procedures and common dentofacial deformities. Zahid et al. ${ }^{17}$ evaluated the psychological and functional status of patients who undergo bimaxillary OS at the Riyadh Military Hospital. The satisfaction of patients after they undergo OS in terms of the quality of life and functional and esthetic improvements should be investigated. Thus, this study aimed to evaluate the satisfaction ratio and the quality of life of patients after OS in terms of function, esthetic appearance of the face, and postoperative complications (swelling and pain). A questionnaire was prepared on the basis of previous studies, along with some modifications to meet the social requirement of the present study.

\section{Materials and Methods}

Owing to the lack in the literature regarding the health-related quality of life after the orthognathic surgery (OS) among patients in Kingdom of Saudi Arabia, this study was conducted. Data of 73 patients who had undergone OS were collected from their medical records and then divided into two parts.

The first part included questions involving the basic demographic information about the patients and postoperative complications, such as swelling, pain, chewing difficulty, and snoring. A number of questions were collected from the World Health Organization and some previous studies to assess the quality of life (WHOQOL). ${ }^{9,10,12,18}$ Those questions were modified to meet the social requirement of the current study.

The second part was introduced as a questionnaire designed by the researchers to measure the patients' satisfaction regarding OS. It consisted of seven questions. ${ }^{11,19}$ The participants were instructed to select one answer from multiple-choice questions related to the satisfaction of the patients after they underwent OS. The questionnaire was related to the appearance of the face from the side and front views and the level of attractiveness perceived by the patients themselves and the opinion of their relatives and friends after OS. The improvements in oral functions (such as eating and chewing) and the overall performance of the oral cavity were assessed.

Data were collected and the questionnaire was answered via a telephone interview with all the patients who underwent OS in Kingdom of Saudi Arabia. The data collected from the patients were pooled for analysis. IBM Statistical Package for the Social Sciences, V 20.1 (SPSS IBM, Inc., Chicago, Illinois, USA) was used for statistical analysis. Descriptive analysis included frequency and percentage, which were calculated for all parameters. The self-satisfaction from each patient in relation to the side, front, and facial appearances, personal judgments about self-attractiveness, and families'/friends' opinion after OS was examined. The improvements in chewing and the general performance of the oral cavity were recorded using a Chi-square test to detect any significant differences. Statistical significance was set at $p>0.05$.

\section{Results}

The data of 73 patients who underwent OS were collected from their medical records. Only 50 patients (response rate was $68.5 \%$ ) completed the telephone interview and the examination [ 28 males (56\%) and 22 females (44\%)]. The age of the patients ranged from
18 years to 50 years, and the mean age was 21.18 years with a standard deviation of 0.717 years. The highest number of subjects [28 patients (56\%)] was found in the young age group of 19-25 years, while the lowest number of subjects [22 patients (44\%)] was in the age group of 46-50 years. Who single patient who was less than 18 years old were recruited (Fig. 1). The results of this study were divided into two parts. The first part was related to functional chewing outcomes, postoperative complication outcome (snoring, pain, swelling), and facial appearances of the patients who underwent OS. Of the patients, 31 (62\%) showed a positive result on snoring, and 8 (16\%) yielded a neutral result after OS (Fig. 2). Almost more than half of the patients (52\%) had a positive response from their surgery in terms of postoperative complications, such as pain and swelling (Fig. 3). Furthermore, 14 patients (28\%) had a neutral response during their recovery time after OS. In terms of the frequency and percentage of the functional chewing outcomes of the patients who underwent OS, 33 (66\%) patients recorded a positive functional chewing outcome, and $10(20 \%)$ yielded a neutral response, indicating a good overall performance among the patients (Fig. 4). High percentages and frequencies were observed in the positive and neutral outcomes of the patients' facial esthetics after OS and registered 27 (54\%) and 13 (26\%), respectively (Fig. 5).

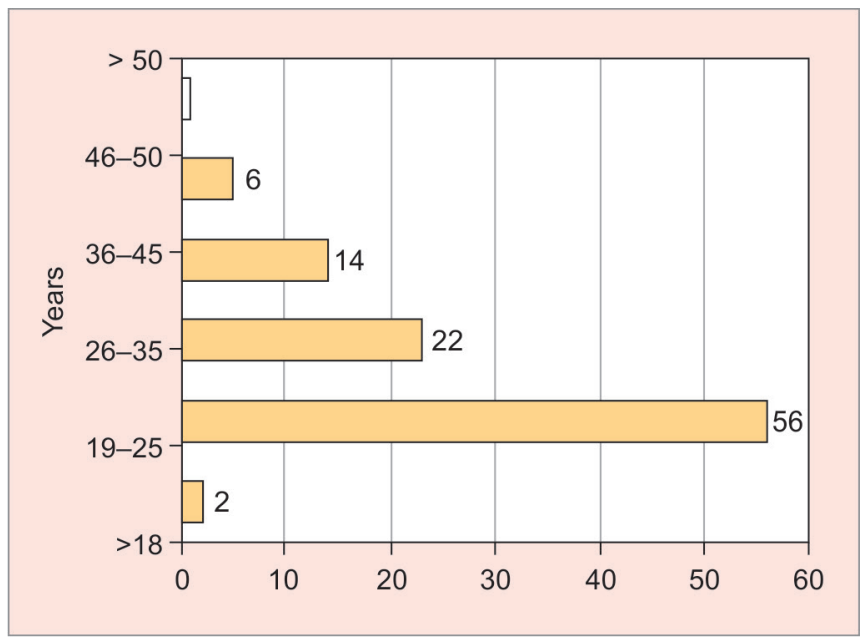

Fig. 1: Percentage of the age groups of patients who underwent OS

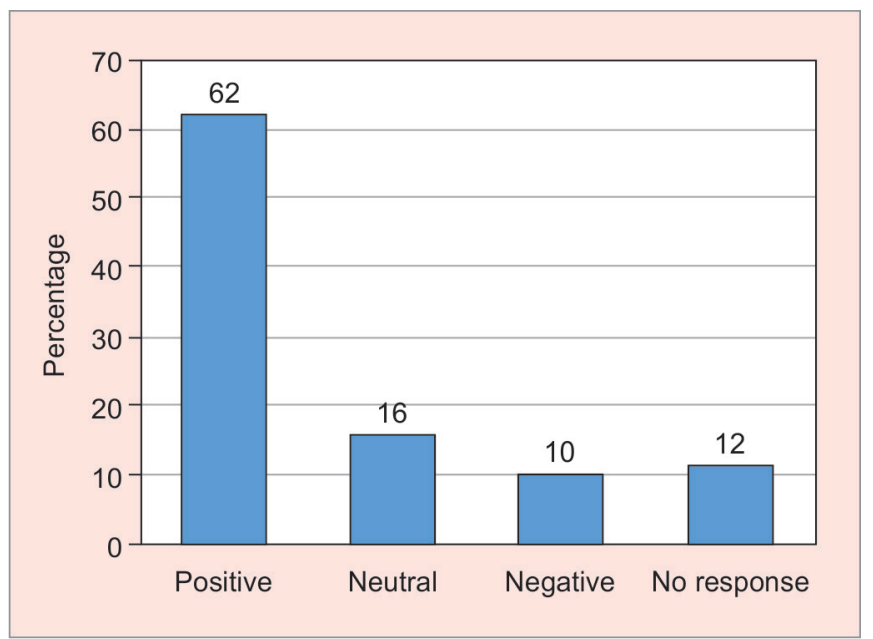

Fig. 2: Percentage of the snoring outcomes of patients who underwent OS 


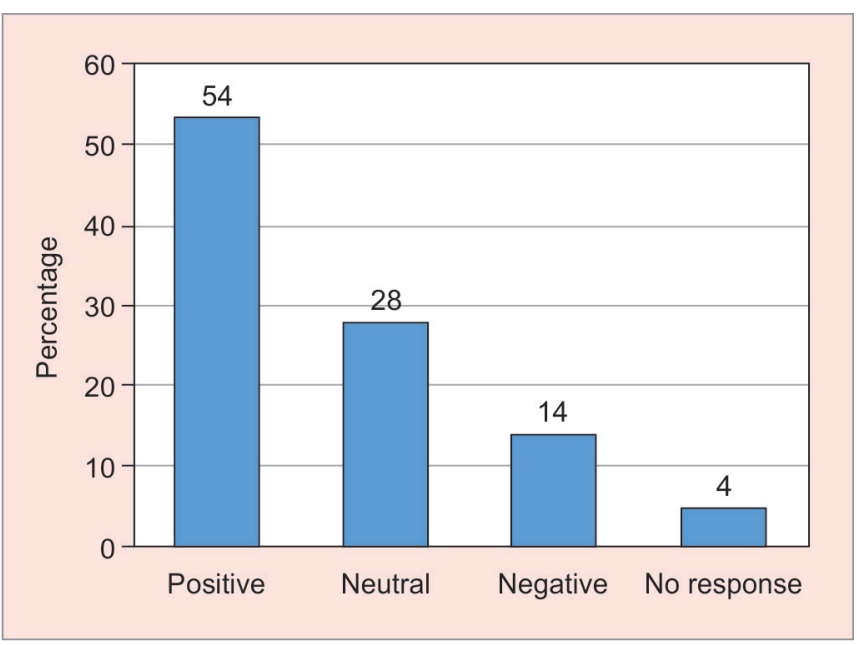

Fig. 3: Percentage of postoperative complication (pain and swelling) outcomes of patients who underwent OS

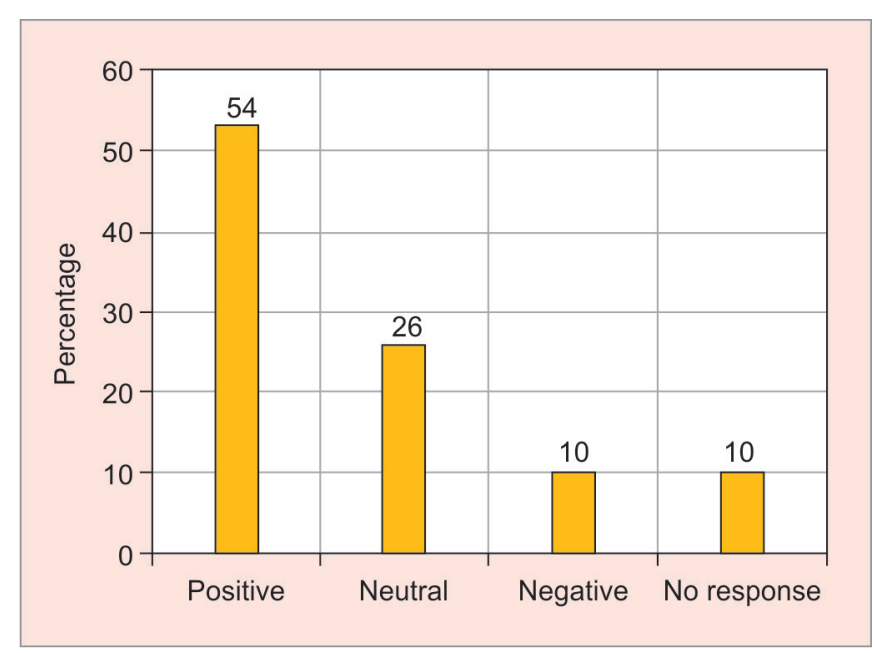

Fig. 5: Percentage of facial esthetic outcomes of patients who underwent OS

The second part was a questionnaire related to the measurement of the patients' satisfaction in relation to the appearance of their face from the side and front views and the opinion about their attractiveness perceived by the patients themselves and their relatives and friends after OS. It also included the ratio of improvements in functions such as eating and chewing and the overall performance of the oral cavity.

The results of the questionnaire answered by the patients via a telephone interview greatly varied. The questions with significant differences were Qs 1 to 3: "Do you like the appearance of your face from the side view and the front view? Are you more conscious of your facial appearance after undergoing OS?" More than $50 \%$ of the patients were significantly satisfied $(p<0.050)$ with their outcomes. Questions 4 and 5 were related to the opinions of the patients' friends and families regarding their facial appearance after OS. The frequencies were between $60 \%$ and $62 \%$ with significant $p=0.043$ and 0.001 . Questions 6 and 7 dealt with the improvements in eating, chewing, and overall oral cavity performance. Only $42-18 \%$ of the participants noticed improvements in their functional activity and overall oral cavity performance $(p=0.033)$, whereas $46-54 \%$ were

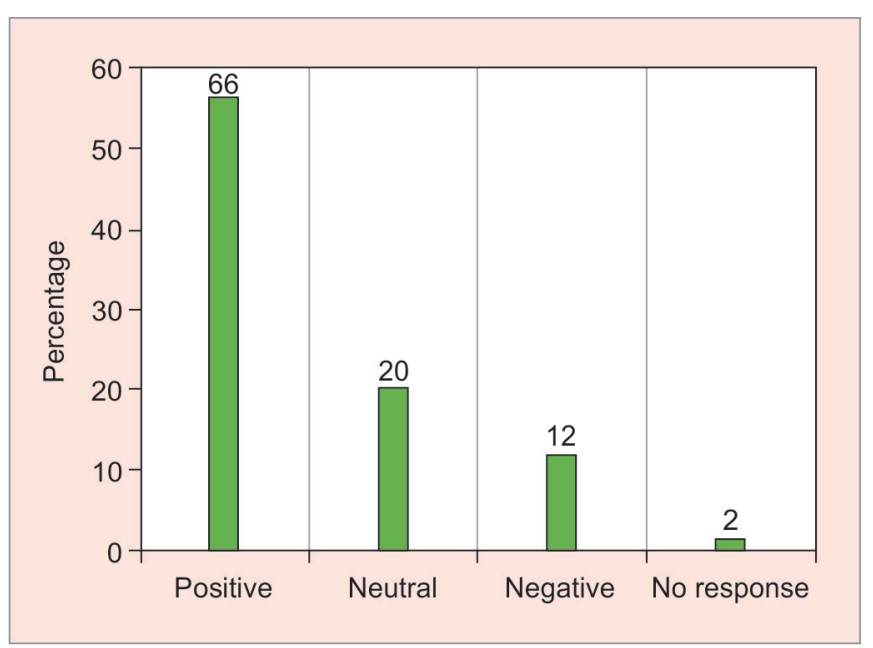

Fig. 4: Percentage of the functional chewing outcome of patients who underwent OS

relatively satisfied in their functional activity and overall oral cavity performance ( $p$ 0.001) (Table 1).

\section{Discussion}

This study investigated the effect of OS on the characteristics of oral health, such as esthetic, functional chewing, pain, and swelling, which were related to the quality of life of the patients who underwent OS. The overall results were satisfactory. The patients yielded more than $60 \%$ of functional chewing outcomes and $54 \%$ of pain, swelling, and facial esthetic outcome of the patients who underwent OS (Figs 3 to 5). These findings were slightly more than the results gained by the previous studies, ${ }^{11,17,20}$ but the values were lesser than those obtained by Al-Asfour et al., ${ }^{21}$ Huang et al., ${ }^{22}$ those founded over $90 \%$, and equal to percentage recorded by Motegi et al., ${ }^{23}$ whose made follow up to 5 years in their postsurgical cases.

The World Health Organization defines the quality of life as the understanding of people in terms of their position in life and relationship with their aims, expectations, standards, and concerns. ${ }^{18}$ With advancements in the medical field, OS positively affects the patient's quality of life.

In the present study, only 50 patients participated, and this value was considered within the acceptable range for a crosssectional study. This finding was consistent with studies mentioned in a systemic review, ${ }^{24}$ even though different types of studies have numerous patients.

Patients should be young because OS mainly deals with esthetics. In this study, the highest percentage of the participants was in the age group between 19 years and 25 years (Fig. 1). This finding was consistent with previous findings. ${ }^{12,17,18,22}$ In a study conducted in Japan, ${ }^{11}$ participants belong to an older age group, ranging from 20 to 50 years.

Figure 2 shows a high percentage (62\%) of positive snoring as a complication after OS. The same finding was reported in a previous study. ${ }^{25}$ Other studies have indicated that the percentage of snoring outcome increases because of certain types and techniques of OS, which may lead to the narrowing of the upper airway at a retropalatal or retroglossal level and trigger snoring in subjects who undergo OS. As such, most surgeons recommend polysomnography before OS. 
Table 1: Percentage of patients' satisfaction

\begin{tabular}{|c|c|c|c|c|c|}
\hline & Questions & Dissatisfied & Relatively satisfied & Satisfied & $p$ value \\
\hline 1 & $\begin{array}{l}\text { Do you like the appearance of your face from the side view after undergoing } \\
\text { orthognathic surgery? }\end{array}$ & 20 & 16 & 54 & $0.049^{*}$ \\
\hline 2 & $\begin{array}{l}\text { Do you like the appearance of your face from the front view after undergoing } \\
\text { orthognathic surgery? }\end{array}$ & 20 & 28 & 52 & $0.049^{*}$ \\
\hline 3 & $\begin{array}{l}\text { After undergoing orthognathic surgery, are you more conscious about your } \\
\text { facial appearance? }\end{array}$ & 10 & 34 & 56 & $0.009^{*}$ \\
\hline 4 & Do you think that orthognathic surgery has made you attractive? & 18 & 10 & 62 & $0.043^{*}$ \\
\hline 5 & $\begin{array}{l}\text { Do you think your family and friends like your facial appearance after you } \\
\text { underwent orthognathic surgery? }\end{array}$ & 30 & 10 & 60 & $<0.001^{*}$ \\
\hline 6 & Does orthognathic surgery improve your eating and chewing abilities? & 12 & 46 & 42 & $0.033^{*}$ \\
\hline 7 & Does orthognathic surgery improve the overall performance of your oral cavity? & 28 & 54 & 18 & $0.001^{*}$ \\
\hline
\end{tabular}

*Significant difference

A high percentage was reported by patients with positive and neutral outcomes regarding functional chewing (Fig. 4). This finding was slightly higher than that recorded in other studies: $35.3 \%{ }^{17}$ and $31 \% .{ }^{20}$ However, this value was equal to the percentage obtained in another study $68.5 \%,{ }^{18}$ but it was quite lower than the percentage reported previously. ${ }^{21}$ In presurgical or postsurgical cases, the value was from $75.8 \%$ to $95 \%$ and $88 \%,{ }^{22}$ but the same percentage was presented in another research. ${ }^{11}$

Postoperative complications were short term, but they persisted for several days and eventually declined or disappeared in terms of intensity. Almost more than $50 \%$ reported swelling and pain as a postoperative complication (Fig. 3). This percentage was equal or slightly less than that recorded in another study ${ }^{12}$ in Riyadh and slightly higher than $50 \%$ on other studies. ${ }^{17,23}$

The relationship between patients' maxillofacial deformities and quality of life after they undergo OS has been extensively studied. The data from the telephone interviews showed that the results from the questionnaires seemed reasonable and provided further important information. ${ }^{11}$ OS significantly changes the patients' facial appearance and life in various ways. This finding was verified in this study, that is, almost $80 \%$ (54\% positive and $26 \%$ neutral). Similar percentages were presented in other studies: $80 \%,{ }^{18} 88 \%,{ }^{12} 79.4 \%{ }^{17}$ for dental appearance and $73.5 \%$ for facial appearance. General facial appearance improvements were also obtained, ${ }^{11,20,23}$ but a high percentage was presented in other studies, which obtained $91.3-96 \% .^{21,22}$

The satisfaction rate of the majority of the patients was high because they were highly motivated with long-standing inner feelings of their esthetic problems. They were concerned about accepting their appearance because surgery should improve the appearance and translate into social/interpersonal changes. ${ }^{12,26}$

The current study also revealed that over $50 \%$ of the patients were satisfied with the appearance of their faces from the side and front views. Even though it was a reasonable percentage, it was the same percentage obtained in another study ${ }^{17}$ in Riyadh and lesser than the percentage recorded in other studies, ${ }^{20,21}$ which showed over $90 \%$ but agreed with those values revealing significant differences gained between variables.

Social attractiveness and relationship strongly affect patients after they undergo OS,22 and they significantly differ ${ }^{18,22,23}$ in their esthetics. Modige et al. stated that most people (friends and families) close to patients after undergoing OS manifested a condition after the operation, but their condition improved, but the same percentages (41.2\%) were recorded by Zahid et al. ${ }^{17}$ This observation was strongly described by the patients undergoing OS during the telephone interview.

Most patients showed a positive effect and a satisfaction rate after they underwent an OS in terms of the overall oral health and satisfaction after OS. This treatment positively influenced the facial esthetic, oral functional, and comfort outcomes after OS. This finding completely agreed with other studies. 11,12,17,20-23

We recommended further studies to compare between genders, preoperative and postoperative OS and included other demographic data, such as the level of education and profession of patients. The type of OS was also not considered. Further longitudinal studies with a long follow-up period are strongly recommended by using international worldwide scales for OS and its impact on the quality of life, such as orthognathic quality of life questionnaire, ${ }^{21}$ different visual analog scales, ${ }^{20,21}$ WHOQOL, ${ }^{11,18,23}$ oral health impact profile assessment, ${ }^{11,22}$ and oral health status questionnaire. ${ }^{23}$

\section{Conclusion}

This cross-sectional study revealed that $62 \%$ of the patients showed a positive result in terms of snoring, and $52 \%$ of the patients had a positive response to postoperative complications, such as pain and swelling, during their recovery time after they underwent OS. Furthermore, $66 \%$ of patients who underwent OS recorded a more positive functional chewing outcome, indicating a good overall performance among patients, and $54 \%$ had positive facial esthetic outcomes. The satisfactory percentage for facial attractiveness from the front and side views was $50 \%$, and the attractiveness increased to $60-62 \%$ according to the opinions of their friends and families. Satisfactory improvements in eating, chewing, and overall oral cavity performance were found to be between $18 \%$ and $42 \%$, and the attractiveness increased to $46-54 \%$ among the relatively satisfied patients.

\section{References}

1. Bellucci CC, Kapp-Simon KA. Psychological considerations in orthognathic surgery. Clin Plast Surg 2007;34:e11-e16. DOI: 10.1016/ j.cps.2007.04.004.

2. Cunningham SJ, Hunt NP. Quality of life and its importance in orthodontics. J Orthod 2001;28:152-158. DOI: 10.1093/ortho/28.2.152.

3. Bennett ME, Phillips CL. Assessment of health-related quality of life for patients with severe skeletal disharmony: a review of the issues. Int J Adult Orthod Orthognath Surg 1999;14:65-75. 
4. Han CW, Yajima Y, et al. Construct validity of the Frenchay activities index for community-dwelling elderly in Japan. Tohoku J Exp Med 2006;210:99-107. DOI: 10.1620/tjem.210.99.

5. Baek SH, Ahn HW, et al. Surgery-first approach in skeletal Class III malocclusion treated with 2-jaw surgery: evaluation of surgical movement and postoperative orthodontic treatment. J Craniofac Surg 2010;21:332-338. DOI: 10.1097/SCS.0b013e3181cf5fd4.

6. Wright EF, North SL. Management and Treatment of Temporomandibular Disorders: A Clinical Perspective. J Man Manip Ther 2009;17(4):247-254. DOI: 10.1179/106698109791352184.

7. Yu HB, Mao LX, et al. The surgery-first approach in orthognathic surgery: a retrospective study of 50 cases. Int J Oral Maxillofac Surg 2015;44:1463-1467. DOI: 10.1016/j.ijom.2015.05.024.

8. Ko EW, Hsu SS, et al. Comparison of progressive cephalometric changes and postsurgical stability of skeletal Class III correction with and without presurgical orthodontic treatment. J Oral Maxillofac Surg 2011;69:1469-1477. DOI: 10.1016/j.joms.2010.07.022.

9. Liou EJ, Chen PH, et al. Surgery-first accelerated orthognathic surgery: orthodontic guidelines and setup for model surgery. J Oral Maxillofac Surg 2011;69:771-780. DOI: 10.1016/j.joms.2010.11.011.

10. Liao YF, Chiu YT, et al. Presurgical orthodontics vs no presurgical orthodontics: treatment outcome of surgical-orthodontic correction for skeletal Class III open bite. Plast Reconstr Surg 2010;126:2074-2083. DOI: 10.1097/PRS.0b013e3181f52710.

11. Modig $M$, Andersson $L$, et al. Patients' perception of improvement after orthognathic surgery: pilot study. Br J Oral Maxillofac Surg 2006;44:24-27. DOI: 10.1016/j.bjoms.2005.07.016.

12. Zahrani AA. Patients satisfaction with orthognathic surgery; a study in a selected Saudi sample. Pakistan Oral Dent J 2004;24(2):129-134.

13. Almasri M, Bukhari SM. Considerations to Planning Orthognathic Surgery in Different Regions of Saudi and the Middle East. Amer J Public Health Research 2014;2(1):6-9. DOI: 10.12691/ajphr-2-1-2.

14. AlBarakati SF, Baidas LF. Orthognathic surgical norms for a sample of Saudi adults: Hard tissue measurements. Saudi Dent J 2010;22: 133-139. DOI: 10.1016/j.sdentj.2010.04.007.

15. Abdullah WA. Changes in quality of life after orthognathic surgery in Saudi patients. Saudi Dent J 2015;27:161-164. DOI: 10.1016/ j.sdentj.2014.12.001.
16. Al Asseri N. Orthognathic surgery: a ten years epidemiological analysis of multi-centers in Riyadh region. Int J Oral Maxillofac Surg 2015;44:e24. DOI: 10.1016/j.ijom.2015.08.435.

17. Zahid M, Al-Qarni M, et al. The Effectiveness of Bimaxillary Osteotomy on the Psychological and Functional Status of Patients. EC Dent Sci 2017;16(6):242-248.

18. Silva ACA, Carvalho RAS, et al. Evaluation of life quality of patients submitted to orthognathic surgery. Dental Press J Orthod 2013;18(5):107-114. DOI: 10.1590/S2176-94512013000500018.

19. Yang L, Yu-dong Xiao Y-D, et al. Does the Surgery-First Approach Produce Better Outcomes in Orthognathic Surgery? A Systematic Review and Meta-Analysis. J Oral Maxillofac Surg 2017;75:2422-2429. DOI: 10.1016/j.joms.2017.06.002.

20. Murphy C, Kearns G, et al. The clinical relevance of orthognathic surgery on quality of life. Int J Oral Maxillofac Surg 2011;40:926-930. DOI: 10.1016/j.ijom.2011.04.001.

21. Al-Asfour A, Waheedi M, et al. Survey of patient experiences of orthognathic surgery: health-related quality of life and satisfaction. Int J Oral Maxillofac Surg 2018;47(6):726-773. DOI: 10.1016/ j.ijom.2017.12.010.

22. Huang CS, Hsu SS, et al. Systematic review of the surgery-first approach in orthognathic surgery. Biomed J 2014;37:184-190. DOI: 10.4103/2319-4170.126863.

23. Motegi E, Hatch JP, et al. Health related quality of life and psychosocial function 5 years after orthognathic surgery. Am J Orthod Dentofacial Orthop 2003;124:138-143. DOI: 10.1016/S0889-5406(03) 00391-3.

24. Soh CL, Narayanan N. Quality of life assessment in patients with dentofacial deformity undergoing orthognathic surgery-A systematic review. Int J Oral Maxillofac Surg 2013;42:974-980. DOI: 10.1016/j.ijom.2013.03.023.

25. Park J-E, Bae S-H, et al. The structural changes of pharyngeal airway contributing to snoring after orthognathic surgery in skeletal class III patients. Maxillofac Plast Reconstr Surg 2017;39:22-30. DOI: 10.1186/ s40902-017-0120-6.

26. Cunningham SJ, Hunt NP, et al. Perceptions of outcome following orthognathic surgery. Br J Oral Maxillofac Surg 1996;34:210-213. DOI: 10.1016/S0266-4356(96)90271-5. 